\title{
Theodor Kocher et César Roux
}

par Christian Bryois

RÉsumé

96 lettres adressées par César Roux (1857-1934) à sa fiancée d'origine russe, Anna Begoune, permettent d'évoquer la formation dans le service de Theodor Kocher du plus prestigieux des chirurgiens vaudois. Sa relation avec Th. Kocher, qu'il compare successivement à Billroth et à R. von Volkmann, est mise en relief.

Dans de nombreuses spécialités médicales, la relève universitaire semble être actuellement difficile. Le Prof.H.Stamm, dans une lettre parue dans le Bulletin des médecins suisses le 28 février 1990, intitulée «A bon entendeur, où est la nouvelle génération de gynécologues?», constate que «trop de professeurs et de chefs de service n'ont plus la capacité ou la volonté de travailler dans l'enseignement et la recherche ni de promouvoir et de former des jeunes pour prendre la relève». Plus loin, il ajoute: «Les professeurs et les médicins-chefs doivent s'acquitter de leur obligation d'encourager la relève, mission que bien des cliniciens aujourd'hui ne remplissent pas parce qu'ils préfèrent opérer eux-mêmes», et enfin, «les professeurs d'universités doivent aussi permettre aux médecins qualifiés d'aller faire leur formation à l'étranger, car ce perfectionnement est nécessaire et urgent».

Nous avons repris ces quelques citations afin d'illustrer parmi les nombreux mérites de Theodor Kocher un de ceux qui nous est apparu comme étant le plus important, à savoir sa grande capacité de former de nombreux et remarquables élèves qui occupèrent des chaires en Suisse et à l'étranger. Nous pouvons citer de Quervain, Matti à Berne, Kummer à Genève, Läng à Amsterdam, Schürch à Bâle, et surtout Cushing et César Roux qui furent parmi ses élèves les plus éminents.

César Roux naquit le 23 mars 1857 dans un petit village du pied du Jura vaudois dénommé Mont-la-Ville. Il est issu d'une famille de réfugiés protestants venant du Val Pragelas, qui s'installa tout d'abord en 1680 dans la région de Bex. Ce n'est qu'en janvier 1871, alors que César Roux a 14 ans, qu'un décret du Grand Conseil vaudois attribua à cette famille la bourgeoisie de Ste-Croix. César était le troisième d'une fratrie de onze enfants, dont sept 
se consacrèrent à l'enseignement. Son père était lui-même instituteur primaire. Quant à sa mère, elle lui donna l'exemple d'une vie imprégnée du sentiment du devoir.

La petite enfance est donc vécue dans des conditions matérielles modestes, imprégnée par la tradition protestante, un environnement rural, son statut d'apatride protestant, ainsi que par une solidarité familiale à toute épreuve.

A 11 ans, il quitte Mont-la-Ville pour Lausanne où il suit le collège classique cantonal. Il entre en 1874 au Gymnase scientifique et obtient son baccalauréat en 1875, ce qui lui permet d'entrer à l'Académie de Lausanne, l'Université de Lausanne n'étant fondée qu'en 1891.

Après quelques hésitations, notamment dues au fait que son père venait de décéder, et grâce à la solidarité familiale, il se détermine pour la carrière médicale, probablement en raison de l'admiration qu'il avait pour un médecin de campagne.

Après avoir passé son premier examen propédeutique à Genève, il décide de poursuivre ses études de médecine dès 1876 à l'Université de Berne. Durant quatre ans, il mènera une vie estudiantine austère, travaillant notamment, en plus de ses études, comme assistant en anatomie pathologique chez Langhans, en physiologie et en anatomie chez Aeby. C'est là que rapidement Theodor Kocher le remarque et lui demande d'exécuter des préparations d'articulations, qui se révélèrent remarquables. Dès lors, Kocher lui propose une place d'assistant après qu'il eut achevé sa thèse de doctorat sur la musculature de l'anus et qu'il eut réussi son examen d'Etat en 1880 .

Contrairement à César Roux, Theodor Kocher est issu d'un milieu aisé et très religieux. Cultivé, il parle couramment l'anglais et le français et a beaucoup voyagé, notamment à Berlin chez von Langenbeck, à Vienne chez Billroth, puis il a visité Paris, l'Angleterre (Spencer Wells) et la Russie. En outre, il entretient des contacts épistolaires étroits avec les principaux chirurgiens allemands, anglais et français. Ce fut certainement un immense privilège pour César Roux que de pouvoir apprendre son métier sous la direction d'un maître dont l'intelligence, les connaissances, la rigueur et l'attitude sereine et réservée impressionnèrent tous les chirurgiens de l'époque, comme le souligne à juste titre le Professeur Sägesser dans son ouvrage consacré à César Roux, son époque et la nôtre.

Kocher enseignait par l'exemple, il parlait peu, était distant, mais s'intéressa particulièrement à César Roux qu'il envoya faire un voyage d'études 
dès avril 1881. Grâce à Mme Jeanne-Marie Allier, petite-fille de César Roux, nous avons pu retrouver, pour la période d'août 1880 à septembre 1884, 96 lettres adressées par César Roux à sa fiancée d'origine russe, Anna Begoune.

A la lecture de ses lettres, on s'aperçoit que durant son voyage d'études, le fait d'être assistant chez Kocher lui ouvre toutes grandes les portes des cliniques de prestigieux chirurgiens germaniques tels que Billroth à Vienne et von Volkmann à Halle. Partout, comme César Roux le relève dans ses écrits, il est fort bien accueilli.

Il séjourne six semaines à Vienne et se montre très intéressé par des pansements au iodoforme, qu'il désire introduire à Berne afin de gagner du temps et améliorer ainsi la qualité de vie des assistants.

Kocher avait la réputation d'être un opérateur précis, méticuleux et minutieux, ainsi que d'être particulièrement attentif aux diagnostics et aux indications opératoires. Bien qu'acquérant une foule de connaissances et une assurance bienvenue, Roux se montre assez critique en comparant Billroth à son maître. Je le cite :

«On m'a beaucoup prévenu contre Billroth, mais j'ai eu beaucoup de plaisir à le voir en clinique. Il est cependant loin d'être aussi bon «Lehrer» que Kocher, il ne se donne pas la peine de faire un diagnostic serré, aussi a-t-il ouvert des abcès multiples des côtes qu'il avait pris pour des sarcomes mous partant du périoste. Cela m'a beaucoup amusé pour une première clinique du semestre».

Et un peu plus loin:

«Il y a deux semaines seulement que je vais chez Billroth, il me plaît beaucoup, c'est vraiment, comme le dit Kocher, sbedeutende Grösses. Mais au point de vue des étudiants, je préfère beaucoup Kocher. Aujourd'hui, j'ai assisté pour la troisième fois à une laparotomie sans qu'on ait fait le diagnostic auparavant».

Quant à l'organisation de la clinique chirurgicale de Billroth, Roux remarque:

«Il y a huit assistants dont deux seulement ont affaire avec une plaie; les autres jouent le rôle de Wärter littéralement: cela consolera ceux de Berne. Ce sont aussi tous des médecins patentés. Billroth a une extrême confiance en son premier et deuxième assistants; lorsqu'il est fatigué, il les laisse terminer l'opération et raconte des histoires, tout en s'essuyant la figure et caressant son gros ventre. Je lui ai vu faire beaucoup de laparotomies et une résection d'estomac au milieu de laquelle il s'en est allé en recommandant à son assistant de veiller à ce qu'elle se termine bien et à ce que la patiente se guérisse».

Bien que trouvant que c'est un plaisir de voir travailler ces messieurs lors des opérations, il ne peut s'empêcher de remarquer: 
«J'ai vu Billroth arracher littéralement une tumeur abdominale chez une femme; il tirait de toutes ses forces sans s'inquiéter d'autre chose que de détacher la tumeur».

Faisant un bilan à la fin de son séjour, Roux note que chaque semaine lui a fait faire des progrès qu'il aurait mis des mois à accomplir s'il était resté à Berne sans avoir voyagé.

«J'y ai appris une toute autre méthode de faire la chirurgie et l'assurance ne me manquera plus».

Mais il ajoute:

«Je me réjouis de retourner chez Kocher que j’apprécie de plus en plus, quoi qu'il m'ait donné des idées assez tranchantes sur beaucoup de points».

Après avoir passé par Prague et Dresde, il se rend à Halle où von Volkmann l'accueille particulièrement bien, lui consacrant notamment beaucoup d'attention et de temps. De son séjour chez Volkmann, il dira:

«J'ai appris une masse de détails pratiques et théoriques que Kocher ne pense pas à nous dire. Cela ne veut pas dire que j'apprécie Volkmann mieux que Kocher, mais je suis très heureux de les voir les deux, je suis beaucoup plus à l'aise avec lui qu'avec Kocher, mais je préfère au fond ce dernier. Il est plus consciencieux vis-à-vis de ses malades et je crois que c'est une qualité très rare et très précieuse chez un chirurgien».

Puis il écrit cette phrase prémonitoire:

«Je puis dire qu'en somme, j'ai tout sacrifié à la chirurgie pendant mon voyage, ai-je bien fait, l'avenir le dira».

Trois semaines après avoir écrit cette phrase, en juin 1882, Roux, à la demande de Kocher, accepte de remplacer Dumont, alors premier assistant, qui se marie. A ce propos, il écrit à sa fiancée Anna Begoune:

«Je veux faire de la chirurgie, et ce n'est pas en faisant un service de 10-12 mois que je pourrais prendre un hôpital avec confiance. C'est pour moi un très lourd sacrifice de voir passer encore tant de temps avant d'entrer dans la pratique, mais mon voyage m'a convaincu que le service un peu long chez Kocher ne peut que m'être précieux, c'est une école comme il n'y en a pas une deuxième, même après avoir vu Billroth et Volkmann. Je reviendrai au pays avec une bonne provision de connaissances nouvelles, mais avec une bonne dose d'admiration de plus pour Kocher».

Si César Roux se montre très admiratif pour Kocher, celui-ci lui manifeste en retour une grande confiance. En effet, Kocher part en vacances durant cinq semaines la veille de la prise de service de César Roux à Berne. Dès lors, Roux profite de l'occasion pour opérer tous les patients pour lesquels il y a une 
indication, tout en se faisant du souci, étant persuadé que Kocher lui fera une remarque sur son trop de zèle. Mais Roux écrit qu’à son retour de vacances, Kocher s'est montré très aimable. Il ajoute:

«Il a, je crois, fait des progrès, il m'a dit que j'avais bien fait d'opérer et a paru complètement d'accord avec les modifications de pansements que je m'étais permises. En somme, il a été avec moi beaucoup plus «vertraulich`qu'auparavant».

En août 1881, César Roux fait quelques commentaires assez vifs sur la construction du nouvel hôpital, démontrant que parfois certains problèmes ont une forte tendance à se répéter, et je fais là allusion à la construction du Centre Hospitalier Universitaire Vaudois (CHUV). Je cite César Roux:

«On travaille activement au nouvel hôpital que, comme Josué [!], je verrai de mes yeux mais où je n'entrerai pas. Imagine-toi que les génies bornés et les vieilles perruques qui dirigent toute l'affaire n'ont pas pensé à des chambres pour les assistants! On les mettrait sous le toit. Gare aux réclamations. Vois-tu ces pauvres diables descendre chaque nuit d'urgence plusieurs fois trois étages avant de savoir si oui ou non il y a du danger et s'ils peuvent retourner au lit. Les oreilles me font mal rien qu'à l'idée des jurons que je pousserais dans un pareil cas. Je suis persuadé que ledit hôpital sera complètement manqué et qu'il coûtera beaucoup plus cher qu'un autre, justement parce qu'on veut absolument économiser».

En juillet 1882, alors que César Roux a décidé de s’installer à Lausanne et qu'il a chargé une de ses sœurs de lui préparer un appartement, il décide de poursuivre sa formation à Berne, notamment après avoir demandé conseil au Dr Niehans qui lui répond catégoriquement qu'il doit rester chez Kocher encore un an et se présenter alors comme spécialiste, prétendant qu'il n'a pas le droit de le faire pour le moment. Cette décision semble aussi favorisée par l'excellente entente qui existe entre César Roux et Theodor Kocher, celui-ci lui laissant souvent beaucoup de responsabilités et une entière liberté pour opérer durant ses absences. En juin 1883, César Roux quitte Berne et décide d'ouvrir au mois de septembre 1883 son cabinet à Lausanne. L'un et l'autre continuèrent d'avoir de bons contacts jusqu'au décès de Theodor Kocher en 1917.

Telle fut la formation médicale de César Roux, qui devint le premier professeur de clinique chirurgicale de l'Université de Lausanne il y a cent ans. Cette formation lui permet de faire œuvre de précurseur dans le cadre de la chirurgie abdominale, étant notamment l'inventeur de l'anse en Y, le premier à avoir pratiqué une oesophagoplastie, ainsi qu'à avoir extirpé un phéochromocytome, et ceci à l'image de son maître Kocher, toujours en se montrant très soucieux et respectueux de ses patients. 
En guise de conclusion, nous reprendrons ce qu'a écrit Henri Vulliet, son élève, dans un hommage pour ses 25 ans d'enseignement de clinique chirurgicale à l'Université de Lausanne:

«De la grande école de Kocher, Roux tient la solide armature et on le sent bien dans son enseignement, mais il s'y joint autre chose de très personnel, d'inimitable, de moins austère, de plus familier. Puis-je me risquer à dire que c'est de la clinique bernoise adaptée à la mentalité vaudoise par un Vaudois».

\section{Bibliographie}

Bonjour, E., Theodor Kocher, Bern (Haupt) 1950.

Clément, G., Le professeur César Roux (1857-1934), Revue méd. Suisse Romande 67 (1935), 257290.

Clément, G., César Roux, l'Homme et le chirurgien, Lausanne (Payot) 1935.

Guisan, A., Taillens, J., Reinhold, P., Nicod, J.-L., Cinquantenaire de la Faculté de Médecine de Lausanne 1890-1940, Lausanne (Roth) 1940.

Reymond, M., Les origines du Professeur Docteur César Roux, Lausanne (Imprimerie vaudoise) 1935.

Saegesser, F., César Roux, son époque et la nôtre, Lausanne (l'Aire) 1989.

Saegesser, F., César Roux (1857-1934) et son époque, Revue méd. Suisse Romande 104 (1984), 403-464.

Stamm, H., To whom it may concern. Wo bleibt der gynäkologische Nachwuchs? Bulletin des médecins suisses 71 (1990), 342-345.

Taillens, J., Decker, P., César Roux, Lausanne (Ed. Eglise nationale vaudoise) 1945.

Vulliet, H., A César Roux, hommage de ses élèves, Revue méd. Suisse Romande 11 (1915), 605615. 


\section{Zusammenfassung}

96 Briefe von César Roux (1857-1934), dem einflussreichsten der Waadtländer Chirurgen, an seine Verlobte, die Russin Anna Begoune, ermöglichen die Vergegenwärtigung seiner Ausbildung bei Theodor Kocher. Sie beleuchten auch sein Verhältnis zu Kocher, den er nacheinander mit Billroth und von Volkmann vergleicht.

\section{Summary}

96 letters addressed by César Roux (1857-1934), the most prestigious surgeon from the Canton of Vaud, to his fiancée, Anna Begoune, native of Russia, allow us to evoke Roux's training in Kocher's departement of surgery. The letters shed light on Roux's relationship with Theodor Kocher, whom he compares successively with Billroth and von Volkmann.

Dr Christian Bryois

Médecin associé

Département Universitaire de

Psychiatrie Adulte

Hôpital de Cery

1008 Prilly 